\title{
GENERALIZED PRANDTL-MEYER WAVES IN RELAXATION HYDRODYNAMICS
}

\author{
BY \\ N. COBURN \\ The University of Michigan
}

1. Introduction. Two topics will be discussed in this paper. First, we shall express the basic relations of the theory of "steady state" relaxation (or nonequilibrium) hydrodynamics in intrinsic forms involving the characteristic variables. The procedure will be similar to that of a previous paper for equilibrium hydrodynamics [1]. Secondly, we shall study these new intrinsic equations and determine when generalized PrandtlMeyer flows exist in nonequilibrium hydrodynamics. The properties of Prandtl-Meyer flows in the steady two-dimensional isentropic nonmagnetic equilibrium supersonic case are discussed by Courant-Friedrichs (cf. p. 265 [2]). Further, the theory of such generalized flows for the corresponding three-dimensional case was given by F. Tan [3]. Finally, the present author discussed a flow of a relativistic and the associated Newtonian fluid for the corresponding nonsteady two-dimensional case [4].

We shall show that: (1) the basic seven equations of steady three-dimensional relaxation hydrodynamics (System I) can be expressed in an intrinsic form involving six dependent variables and curvatures of three congruences of curves (System IIIa); (2) one of the relations of System IIIa is a linear combination of the other six relations (Theorem 3); (3) if this relation is omitted, then the resulting system of equations (System IIIb) is equivalent to System I or IIIa. Then, we shall introduce a coordinate system in which a family of planes, the characteristics, that envelope a right cylinder are coordinate surfaces. The bicharacteristics are a family of lines in each characteristic plane. Sec. 3 is devoted to determining the form of the equations of System IIIb in this coordinate system. Finally, simple waves of Cases I and IIa, b (cf. p. 121 [5]) are defined for the case of steady "supersonic" three-dimensional relaxation hydrodynamics. In both Case I and IIa, the bicharacteristics are parallel lines in the above family of characteristic planes; in Case IIb, the bicharacteristics form a family of radial lines in each characteristic plane. However, by definition: in Case I, all the flow variables are $C^{1}$ functions of the density, $\rho$; in Cases IIa and IIb, except for the relaxation scalar, $K$, and the relaxation rate variable, $\bar{q}$, all the remaining flow variables are $C^{1}$ functions of the density, and of the density and entropy, respectively. It is shown that the flows of both cases must be generalized Prandtl-Meyer flows. Further, in Case I, the flow is isentropic of the "equilibrium type" (cf. p. 127 [5]), and such that $K$ depends on a special function of $\rho$ (Theorem 9). In Case IIa, the flow is nonisentropic of a particular nonequilibrium type, and such that $K / \bar{q}$ is a particular function of $\rho$ (Theorem 10 , cf. p. 124 [5]). Finally, sufficient conditions for a chemically reacting gas to possess simple waves of Case I are determined.

2. Intrinsic forms of the basic relations. Let $T, \rho, q, S, p, v_{i}(j=1,2,3)$ denote the

Received November 9, 1965. This work was done under a grant (20-63) of the Air Force Office of Scientific Research, administered by the University of Michigan Office of Research Administration. 
temperature, density, relaxation variable, entropy, pressure, velocity vector, respectively. Further, let $x^{j}(j=1,2,3)$ be a Cartesian orthogonal coordinate system and let

$$
\partial_{i} \equiv \partial / \partial x^{i} .
$$

Although there is no difference between contravariant and covariant vectors in Cartesian orthogonal coordinates, we shall introduce both quantities in order to use the Einstein summation convention of summing on any repeated upper and lower index (see also the coordinate system of Sec. 3). Now, we define the variable, $\bar{q}$, by

$$
\bar{q} \equiv v^{i} \partial_{i} q .
$$

Also, we introduce two more scalar variables: the relaxation scalar, $K$, and the internal energy, $e$. Then, the equations of motion, continuity, and energy for the steady state flow of a compressible, nonmagnetic fluid in relaxation hydrodynamics are [5]

$$
\begin{gathered}
\rho v^{k} \partial_{k} v_{i}+\partial_{i} p=0, \\
\rho \partial_{i} v^{i}+v^{i} \partial_{i} \rho=0, \\
K T v^{i} \partial_{i} S=\bar{q}^{2}, \\
\bar{q}=-K \partial e / \partial q .
\end{gathered}
$$

It should be noted that the above system contains three independent thermodynamical variables. If we choose these variables to be $\rho, q, S$, then in one important case [5], we can assume $T, p, e, K, \bar{q}$ are $C^{1}$ functions of $\rho, S, q$. Further, by use of a proper formulation of the first law of nonequilibrium thermodynamics [6], it can be shown that coefficients $A, B, C$ exist so that

$$
\begin{gathered}
A \equiv \frac{\partial p}{\partial \rho}=2 \rho \frac{\partial e}{\partial \rho}+\rho^{2} \frac{\partial^{2} e}{\partial \rho^{2}}, \\
B \equiv \frac{\partial p}{\partial S}=\rho^{2} \frac{\partial^{2} e}{\partial S \partial \rho}, \\
C \equiv \frac{\partial p}{\partial q}=\rho^{2} \frac{\partial^{2} e}{\partial \rho \partial q} .
\end{gathered}
$$

Finally, we define the coefficients $F, G$ by

$$
F \equiv \partial^{2} e / \partial q \partial S, \quad G \equiv \partial^{2} e / \partial q^{2} .
$$

Now, we replace (2.5) by an equation involving only derivatives with respect to $x^{i}$. To do so, we form the total derivative of (2.5) and find by use of (2.5), (2.8), and (2.9) that

$$
v^{i} \partial_{i} \bar{q}=-K\left(\frac{C}{\rho^{2}} v^{i} \partial_{i} \rho+F v^{i} \partial_{j} S+G v^{i} \partial_{i} q\right)+\frac{\bar{q}}{K} v^{i} \partial_{i} K
$$

The system (2.1)-(2.4), (2.10) consists of seven partial differential equations in the seven dependent variables $\bar{q}, q, \rho, S, v_{i}$. Note that: (1) $\bar{q}$ is defined in terms of these variables and their derivatives by $(2.1)$; (2) this system differs from the corresponding equilibrium hydrodynamics system in that the relation (2.4) is not quasi-linear; (3) the pressure, $p$, can be eliminated from (2.2) by noting that $p$ is a $C^{1}$ function of $\rho, q, S$ 
and using the coefficients $A, B, C$ of (2.6)-(2.8) to obtain

$$
\partial_{i} p=A \partial_{i} \rho+B \partial_{i} S+C \partial_{i} q .
$$

Definition 1. The equations (2.1), (2.2)-(2.4), and (2.10) will be called System I.

Our next step is to introduce the characteristic manifolds [5] of (2.2)-(2.4), and (2.10). Let $\phi\left(x^{i}\right)=\bar{c}$, where $\bar{c}$ is a constant, denote a family of such manifolds. Then, the unit normal vector to the characteristics, $n_{i}$, is determined by (cf. (2.48), (2.51) of [5])

$$
R\left(v^{i} n_{i}\right)^{2}=Z
$$

where $R, Z$ are defined by ( $k$ is a $C^{1}$ function of $\phi$ )

$$
\begin{gathered}
R \equiv F(2 \bar{q}+k K)+G T K, \\
Z \equiv F(2 \bar{q}+k K) c_{\infty}^{2}+G T K c_{0}^{2} .
\end{gathered}
$$

Note, the limit speeds $c_{0}, c_{\infty}$ are (cf. (2.47) of [5])

$$
c_{0}^{2} \equiv A-\frac{C^{2}}{\rho^{2} G}, \quad c_{\infty}^{2} \equiv A-\frac{B C}{\rho^{2} F} .
$$

From (2.12), we obtain in the "supersonic" case (where the normal cone is real)

THEOREM 1. In the case of nonequilibrium flow (as in equilibrium flow [7]), the normal and the associated bicharacteristic cones at any point $P$ are right circular cones with the velocity vector along the axis.

Thus, if $t^{i}$ is a unit vector along the bicharacteristic corresponding to $n^{i}$, we may write (cf. p. $57[7]$ )

$$
v^{i}=c n^{i}+b t^{i} .
$$

Here, $v$ is the magnitude of $v^{i}$ and $c, b$ are defined by

$$
c^{2} \equiv \frac{Z}{R} ; \quad b^{2} \equiv v^{2}-c^{2} .
$$

Now, we introduce the directional derivatives along $n^{i}, t^{i}$ and the unit vector $\bar{p}^{i}$, where $\bar{p}^{i}$ is orthogonal to both $t^{i}, n^{i}$, and is such that the ordered triad $t^{i}, \bar{p}^{i}, n^{i}$ form a right hand set. We shall use the notation of a previous paper [1] and write

$$
\frac{\partial}{\partial t} \equiv t^{i} \partial_{i} ; \quad \frac{\partial}{\partial n} \equiv n^{i} \partial_{j} ; \quad \frac{\partial}{\partial \bar{p}} \equiv \bar{p}^{i} \partial_{i}
$$

Replacing $v^{i}$ of (2.1)-(2.4), (2.10) by (2.16) and also replacing $\partial_{i} p$ by (2.11), we find after scalar multiplication of $(2.2)$ by $t^{j}, n^{i}, \bar{p}^{i}$, respectively

$$
\begin{gathered}
c \frac{\partial q}{\partial n}+b \frac{\partial q}{\partial t}=\bar{q} \\
\rho b \frac{\partial b}{\partial t}+\rho c^{2} t^{i} n^{k} \partial_{k} n_{i}+\rho c \frac{\partial b}{\partial n}+\rho b c t^{i} t^{k} \partial_{k} n_{i}=-A \frac{\partial \rho}{\partial t}-B \frac{\partial S}{\partial t}-C \frac{\partial q}{\partial t} \\
\rho c \frac{\partial c}{\partial n}+\rho c b n^{i} n^{k} \partial_{k} t_{i}+\rho b \frac{\partial c}{\partial t}+\rho b^{2} n^{i} t^{k} \partial_{k} t_{i}=-A \frac{\partial \rho}{\partial n}-B \frac{\partial S}{\partial n}-C \frac{\partial q}{\partial n} \\
\rho c^{2} \bar{p}^{i} n^{k} \partial_{k} n_{i}+\rho c b \bar{p}^{i} n^{k} \partial_{k} t_{i}+\rho c b \bar{p}^{i} t^{k} \partial_{k} n_{i}+\rho b^{2} \bar{p}^{i} t^{k} \partial_{k} t_{i}=-A \frac{\partial \rho}{\partial \bar{p}}-B \frac{\partial S}{\partial \bar{p}}-C \frac{\partial q}{\partial \bar{p}^{2}}
\end{gathered}
$$




$$
\begin{gathered}
\rho c \partial_{i} n^{i}+\rho b \partial_{i} t^{i}+\rho \frac{\partial c}{\partial n}+\rho \frac{\partial b}{\partial t}+c \frac{\partial \rho}{\partial n}+b \frac{\partial \rho}{\partial t}=0 \\
K T\left(c \frac{\partial S}{\partial n}+b \frac{\partial S}{\partial t}\right)=\bar{q}^{2}
\end{gathered}
$$

$c \frac{\partial \bar{q}}{\partial n}+b \frac{\partial \bar{q}}{\partial t}+K C \rho^{-2}\left(c \frac{\partial \rho}{\partial n}+b \frac{\partial \rho}{\partial t}\right)-\frac{\bar{q}}{K}\left(c \frac{\partial K}{\partial n}+b \frac{\partial K}{\partial t}\right)=-F T^{-1} \bar{q}^{2}-G K \bar{q}$,

The system (2.19)-(2.25) consists of seven equations in the six dependent variables $\rho, S$, $q, \bar{q}, b, c$. In addition, these equations contain the directional derivatives of $b, c$ and the curvatures of the $t^{i}, n^{i}, \bar{p}^{i}$ congruences of curves.

To show the manner in which these curvatures enter the theory, we note that if $m^{i}, u^{i}, w^{i}$, are the curvature vectors of the $t^{i}, n^{i}, \bar{p}^{i}$ congruences, respectively, then by definition

$$
\begin{aligned}
m^{i} & \equiv t^{k} \partial_{k} t^{i}, \\
u^{i} & \equiv n^{k} \partial_{k} n^{j}, \\
w^{i} & \equiv \bar{p}^{k} \partial_{k} \bar{p}^{i} .
\end{aligned}
$$

Further, since $n^{i}$ is a unit vector which is orthogonal to a family of surfaces, the characteristics, we may write (cf. p. 28 [8])

$$
\partial_{j} n_{k}=s_{j k}+n_{j} u_{k}
$$

where $s_{j k}$ is the symmetric second fundamental tensor of the characteristic surfaces. An examination of (2.23) will show that the geometric significance of

$$
\partial_{j} t^{i}, \quad \partial_{i} n^{i}
$$

must be determined. From the well known decomposition of the metric tensor, $g^{i k}$, we find (cf. p. 96, [9])

$$
g^{i k}=t^{i} t^{k}+n^{i} n^{k}+\bar{p}^{i} \bar{p}^{k}
$$

Thus, we obtain

$$
\partial_{j} t^{i}=g^{i k} \partial_{j} t_{k}=n^{i} n^{k} \partial_{j} t_{k}+\bar{p}^{i} \bar{p}^{k} \partial_{j} t_{k}
$$

In view of the orthogonality relations between $t^{i}, n^{i}, \bar{p}^{i}$, and (2.26) we see that

$$
\begin{aligned}
& n^{i} n^{k} \partial_{i} t_{k}=-t^{k} u_{k}, \\
& \bar{p}^{i} \bar{p}^{k} \partial_{j} t_{k}=-t^{k} w_{k} .
\end{aligned}
$$

Thus, (2.29) becomes

$$
\partial_{j} t^{i}=-t^{i}\left(u_{i}+w_{j}\right) .
$$

Further, by use of (2.27) we find

$$
\partial_{j} n^{i}=g^{j k} s_{j k}=M^{*}
$$

where $M^{*}$ is the mean curvature of the characteristic surfaces.

Definition 2. The geometric relations (2.26), (2.27), (2.30)-(2.32) will be called the System II. 
By use of System II, the relations (2.20)-(2.23) can be expressed in the forms

$$
\begin{gathered}
\rho b \frac{\partial b}{\partial t}+\rho c \frac{\partial b}{\partial n}+\rho c^{2} t^{i} u_{i}-\rho b c n^{i} m_{i}=-A \frac{\partial \rho}{\partial t}-B \frac{\partial S}{\partial t}-C \frac{\partial q}{\partial t}, \\
\rho c \frac{\partial c}{\partial n}+\rho b \frac{\partial c}{\partial t}-\rho c b t^{i} u_{i}+\rho b^{2} n^{i} m_{i}=-A \frac{\partial \rho}{\partial n}-B \frac{\partial S}{\partial n}-C \frac{\partial q}{\partial n}, \\
\rho c^{2} \bar{p}^{i} u_{i}+\rho b^{2} \bar{p}^{i} m_{j}+\rho c b\left(\bar{p}^{i} n^{k} \partial_{k} t_{i}+\bar{p}^{i} t^{k} \partial_{k} n_{i}\right)=-A \frac{\partial \rho}{\partial \bar{p}}-B \frac{\partial S}{\partial \bar{p}}-C \frac{\partial q}{\partial \bar{p}}, \\
\frac{\partial(c \rho)}{\partial n}+\frac{\partial(b \rho)}{\partial t}+\rho c M^{*}-\rho b\left(u^{i}+w^{i}\right) t_{i}=0 .
\end{gathered}
$$

Definition 3a. The relations (2.19), (2.24), (2.25), (2.33)-(2.36) will be called the System IIIa.

Definision 3b. The equations (2.19), (2.24), (2.25), (2.33), (2.35) and (2.36) will be called the System IIIb.

We can summarize our results by

Theorem 2. The equations of System IIIa and the relation (2.16) (which defines $\left.t^{i}, n^{i}\right)$ are equivalent to the basic relations of relaxation hydrodynamics-the equations of the System I [(2.1)-(2.4), (2.10)].

Proof. The equivalence of these two systems follows from the relation for any scalar, $Q$ (see (2.16))

$$
v^{i} \partial_{i} Q=c \frac{\partial Q}{\partial n}+b \frac{\partial Q}{\partial t}
$$

and the equations of System II.

The relations (2.1)-(2.4), (2.10) of System I or the equivalent relations of System IIIa, are of three types: (1) the scalar, $\bar{q}$, is defined by (2.19); (2) the relations (2.20)(2.24) are equivalent to the laws of motion, continuity, and one of the energy conditions, respectively; (3) the relation (2.25) constitutes a new assumption. That is, the relation (2.10) (which is equivalent to (2.25)) is a necessary condition for the second energy condition (2.5) but is not sufficient for the validity of (2.5). In fact, it appears that two additional relations of this type (formed by letting $w^{i} \partial_{i}, u^{i} \partial_{i}$ operate on (2.5), where $v^{i}, w^{i}, u^{i}$ are mutually orthogonal) are needed. This would lead to an overdetermined system of nine equations in six dependent variables.

This surplus of relations is deceptive. Thus, if $K(\rho, S, q)$ and $e(\rho, S, q)$ (or $\partial e / \partial q$ ) are known functions of their arguments then (2.5) can be used to determine $\bar{q}$. When these functions are unknown but $C, F, G$ are known then we replace (2.5) by (2.10) and obtain a relation of System I. Further, we consider only those solutions of System I which satisfy (2.5). That is, (2.5) becomes part of the boundary data.

Now, we shall show that the basic system is determinate when the internal energy of a non-equilibrium fluid is properly defined.

Theorem 3. If the internal energy of a nonequilibrium fluid, $e(\rho, S, q)$, is defined to be the sum of the kinetic and deformation energies, as in an equilibrium fluid (cf. p. 269 [8], then the relations (2.2)-(2.5) of System I or (2.24), (2.25), (2.33), (2.34), (2.36) of System IIIa are linearly dependent for a nonheat conducting fluid (cf. p. 957 [6]). 
Proof. Let us define $e$ by (cf. p. 269 [8])

$$
\frac{d e}{d t} \equiv \frac{\rho}{2} \frac{d\left(v^{2}\right)}{d t}+\partial_{i}\left(p v^{j}\right)
$$

where $d / d t$ is defined as

$$
\frac{d}{d t} \equiv v^{i} \partial_{i}
$$

By use of the first law of thermodynamics for a nonequilibrium fluid and (2.4), (2.5), we find (cf. p. 957 [6]) that the rate of loss of internal energy per unit volume $d e / d t$ (which is converted to kinetic and potential energy) is

$$
\frac{d e}{d t}=\frac{-p}{\rho} \frac{d \rho}{d t}
$$

expanding the second term in the right hand side of the identity (2.38), we obtain

$$
\frac{d e}{d t} \equiv \frac{\rho}{2} \frac{d\left(v^{2}\right)}{d t}+p \partial_{j} v^{i}+v^{i} \partial_{i} p .
$$

If we multiply the equations of motion $(2.2)$ by $v^{j}$, we find

$$
0=\frac{\rho}{2} \frac{d\left(v^{2}\right)}{d t}+v^{i} \partial_{i} p
$$

Multiplying the equation of continuity $(2.3)$ by $\left(p \rho^{-1}\right)$, we obtain

$$
0=p \partial_{j} v^{i}+\frac{p}{\rho} v^{i} \partial_{j} \rho .
$$

By adding the corresponding right and left sides of (2.40), (2.42a) and (2.42b), we obtain the identity (2.41). This verifies the linear dependence of (2.2)-(2.5) of System I. Since (2.42a) can be obtained by multiplying (2.33), (2.34) by b, c, respectively, and adding the resulting equations, the linear dependence of $(2.24),(2.25),(2.33),(2.34),(2.36)$ of System IIIa follows directly.

From Theorems 2, 3 and Definition 3, we find

TheOREm 4. The relations of System IIIb (see Definition $3 \mathrm{~b}$ ) form a system of six equations in the six dependent variables $c, b, \rho, S, q, \bar{q}$ (the modified System IIIa) which is equivalent to the original System I.

Proof. The verification of this result stems from the following facts about (2.34): (1) this is an equation of System IIIa (cf. Definition 3a); (2) this equation depends linearly on (2.19), (2.24), (2.33) and (2.36) of System IIIb (cf. Theorem 3); (3) the System IIIa is equivalent to System I (cf. Theorem 2).

Because of Theorem 4, we shall consider only System IIIb or (2.19), (2.24), (2.25), (2.33), (2.35) and (2.36) in our future work.

By a similar procedure to that used in determining System IIIa, we find that the components of the vorticity vector (where $e^{i p Q}$ is the permutation tensor density)

$$
\omega^{i}=e^{i p q} \partial_{p} v_{a}
$$

are (cf. p. $240[1]$ ) 


$$
\begin{gathered}
\omega^{i} t_{i}=c \bar{p}^{k} u_{k}-\frac{\partial c}{\partial \bar{p}}+b\left(n^{i} \bar{p}^{k}-n^{k} \bar{p}^{j}\right) \partial_{i} t_{k}, \\
\omega^{i} n_{i}=\frac{\partial b}{\partial \vec{p}}-b \bar{p}^{k} m_{k}, \\
\omega^{i} \bar{p}_{i}=\frac{\partial c}{\partial t}-\frac{\partial b}{\partial n}-s_{i k} t^{k} t^{i}-c t_{k} u^{k} .
\end{gathered}
$$

3. A special class of flows in relaxation hydrodynamics. In this section, we study the case where: (1) the characteristic surfaces consist of a family of planes parallel to the parallel lines $\left(x^{3}=\right.$ variable); (2) the bicharacteristics are a family of lines in any characteristic plane. Following a procedure similar to that of Tan [3], we assume that the family of characteristic planes with unit normal vector $n^{i}$, envelope a right cylinder $C$, whose generators are parallel to the $x^{3}=$ variable coordinate lines of the orthogonal Cartesian coordinates $x^{i},(j=1,2,3)$. Let $C$ intersect $x^{3}=0$ in the curve $\bar{C}$ and let $*^{*} s^{i}$ be the unit tangent vector to $\bar{C}$. Further let $C^{*}$ be a specific orthogonal trajectory of the lines $L$ tangent to $\bar{C}$, (i.e. $C^{*}$ is an involute of $\bar{C}$ (cf. p. 30 [10])). Now if $\theta$ denotes the angle between any line $L$ and the $x^{1}$-axis (that is, the line $x^{2}=0$, lying in the plane $x^{3}=0$ ), then we can assume $* s^{i}(\theta)$ is of class $C^{1}$ in $\theta$. Evidently, $* s^{j}$ lies along the lines $L$. Again, if $s(\theta)$ denotes the arc length along $\bar{C}$ from a point $O^{\prime}$ to a point $Q$ where $L$ is tangent to $\bar{C}$, then for any constant, $\alpha$, the distance

$$
Q P^{*}=\alpha-s
$$

determines a point $P^{*}$ where an orthogonal trajectory, $C^{*}$, intersects $L$ (cf. p. 31 [10]).

We shall consider an orthogonal coordinate system where the coordinate surfaces are: (1) the planes, $z={ }^{\prime} x^{3}=$ constant; (2) the planes, $\theta={ }^{\prime} x^{2}=$ constant, which envelope the right cylinder, $C$; (3) the right cylinders, $\alpha={ }^{\prime} x^{1}=$ constant, which intersect any plane, $z={ }^{\prime} x^{3}=$ constant, in an orthogonal trajectory of the lines $L^{\prime}$, which lie in $z=$ constant and are parallel to $L$. The vectors $*^{i}, n^{i}, k^{i}$ are the unit vectors along the $\alpha=$ variable, $\theta=$ variable, $z=$ variable coordinate lines, respectively, and oriented so that for positive sensed $* s^{i}, n^{i}, k^{i}$, the variables $\alpha, \theta, z$, respectively, are increasing. It follows that the ordered triad $* s^{i}, n^{i}, k^{i}$ forms a right handed system.

In the $\alpha, \theta, z$ coordinates, the element of are is

$$
d s^{2}=(\bar{A} d \alpha)^{2}+(\bar{B} d \theta)^{2}+(\bar{C} d z)^{2}
$$

where, since $z$ and $\alpha$ measure distance (see (3.1)),

$$
\bar{A}=\bar{C}=1 .
$$

Theorem 5. The metric coefficient $\bar{B}$ of $(3.2)$ is

$$
\bar{B}=\alpha-s=R^{*}
$$

where $R^{*}$ is the radius of curvature of $C^{*}$ at $P^{*}$.

Proof. Since $Q P^{*}$ of (3.1) is normal to the involute $C^{*}$ of $\bar{C}, \bar{B}$ must be the radius of curvature of $C^{*}$ at $P^{*}$. From a well known result (cf. p. 31 [10]), we find for a plane curve $\bar{C}$ that $\bar{B}$ is given by (3.4) (where $s(\theta)$ is known when $\bar{C}$ is specified).

From (3.3), (3.4), the Christoffel symbols of the first and second kind (cf. p. 17 [9]) are easily computed. First, we find the metric tensor for ' $x^{1}=\alpha,{ }^{\prime} x^{2}=\theta,{ }^{\prime} x^{3}=z$. Use 
of (3.3), and (3.4) leads to

$$
\begin{array}{lll}
{ }^{\prime} g_{11}={ }^{\prime} g_{33}=1 ; & \prime g_{22}=(\alpha-s)^{2}, & \\
{ }^{\prime} g^{11}=g^{33}=1 ; & g^{33}=0 \\
g^{22}=(\alpha-s)^{-2}, & { }^{i j} g^{i j}=0
\end{array}
$$

where $i \neq j$. The only nonvanishing Christoffel symbols are:

$$
\begin{gathered}
-\left[\begin{array}{c}
22 \\
1
\end{array}\right]=\left[\begin{array}{c}
12 \\
2
\end{array}\right]=\alpha-s, \quad\left[\begin{array}{c}
22 \\
2
\end{array}\right]=-(\alpha-s) s^{\prime} \\
\Gamma_{12}^{2}=(\alpha-s)^{-1}, \quad \Gamma_{22}^{2}=(\alpha-s)^{-1} s^{\prime}, \quad \Gamma_{22}^{1}=-(\alpha-s)
\end{gathered}
$$

where primes denote derivatives with respect to $\theta$, that is

$$
s^{\prime} \equiv \frac{d s}{d \theta} .
$$

The $(\alpha, \theta, z)$ components of $t^{i}, * s^{i}, k^{i}, \bar{p}^{i}, n^{i}, t_{i}$, are

$$
\begin{gathered}
t^{i}(\bar{a}, 0, \bar{b}) ; \quad{ }^{*} s^{i}(1,0,0) ; \quad k^{i}(0,0,1), \\
\bar{p}^{i}(\bar{b}, 0,-\bar{a}) ; \quad n^{i}\left[0,(\alpha-s)^{-1}, 0\right], \\
t_{i}(\bar{a}, 0, \bar{b})
\end{gathered}
$$

where if $\psi$ is the angle between $t^{i}$ and $* s^{i}$

$$
\bar{a}=\cos \psi, \quad \bar{b}=\sin \psi
$$

The ordered triad $t^{i}, \bar{p}^{i}, n^{i}$ have been ordered so that they form a right hand system.

With the aid of (3.3)-(3.11), the intrinsic forms (where $\nabla_{i}$ denotes the covariant derivative)

$$
\frac{\partial c}{\partial t}, \frac{\partial c}{\partial \bar{p}}, \frac{\partial c}{\partial n}, u_{i}, \bar{p}^{i} n^{k} \nabla_{k} t_{i}, \bar{p}^{k} n^{i} \nabla_{k} t_{i}
$$

can be evaluated. We find by use of (3.10)

$$
\begin{aligned}
& \frac{\partial c}{\partial t}=\bar{a} \frac{\partial c}{\partial \alpha}+\bar{b} \frac{\partial c}{\partial z}, \\
& \frac{\partial c}{\partial \bar{p}}=\bar{b} \frac{\partial c}{\partial \alpha}-\bar{a} \frac{\partial c}{\partial z}, \\
& \frac{\partial c}{\partial n}=(\alpha-s)^{-1} \frac{\partial c}{\partial \theta} .
\end{aligned}
$$

Two types of families of straight line bicharacteristics will be considered: (1) bicharacteristics in each characteristic plane form a family of parallel lines and hence the angle $\psi$ of (3.11) is a $C^{1}$ function of only $\theta$; (2) the bicharacteristic in each plane $M$ form a family of lines all of which pass through the point $Q$ of the curve $\bar{C}$ and hence the angle $\psi$ of (3.11) is a $C^{1}$ function of $\theta$ and $z /(\alpha-s)$, as $Q P^{*}$ is $(\alpha-s)$ by (3.1). Thus, we define the two families of bicharacteristics by

Definition 4. Case a will consist of bicharacteristics determined by $t^{j}$ of (3.10) where $\psi=\psi(\theta)$; Case $\mathrm{b}$ will be such that $t^{i}$ of $(3.10)$ have $\psi=\psi(\theta, w)$ where

$$
w=\frac{z}{\alpha-s} .
$$


A further generalization of Case b can be derived by replacing $Q$ by any point $Q^{*}$ which is obtained by translating the line $Q P^{*}$ through a distance $z(\theta)$ parallel to the $z$-axis, where $z(\theta)$ is an arbitrary $C^{1}$ function of $\theta$. Then $(3.12 \mathrm{~b})$ must be replaced by

$$
w=\frac{z-z(\theta)}{\alpha-s} .
$$

Evidently, Case a is a special subcase of Case b. Since $n^{1}$ is orthogonal to $\bar{C}$ at $P^{*}$, from: (1) the Frenet-Serret formulas for $C^{*}$; (2) the relation $(3.4)$; (3) the fact that the ordered triad $*^{i}, n^{i}, k^{i}$ forms a right hand system; (4) the component relation for $* s^{i}$ in (3.10), it follows that for $\alpha>s$

$$
u^{i}:-(\alpha-s)^{-1}(1,0,0) \text {. }
$$

Further, since in Case a, $\bar{p}^{i}, t^{i}$ are congruences of straight lines, we see that

$$
m^{i}=w^{i}=0 .
$$

Finally, by use of (3.7), (3.8), and (3.10) and a direct but lengthy computation, we find for both Cases a and $b$

$$
\begin{gathered}
\bar{p}^{j} n^{k} \nabla_{k} t_{i}=-(\alpha-s)^{-1} \frac{\partial \psi}{\partial \theta}, \\
\bar{p}^{k} n^{i} \nabla_{k} t_{i}=0 .
\end{gathered}
$$

For Case b, (3.14a) must be replaced by

where

$$
m^{i}=0, \quad w^{i}=k t^{i}
$$

$$
k \equiv-\left[z^{2}+(\alpha-s)^{2}\right]^{-1 / 2} .
$$

Thus, both Cases a and b can be treated simultaneously by: (1) introducing the curvature $k$ and allowing $k$ of Case $\mathrm{b}$ to vanish for Case a; (2) assuming $\psi$ is a $C^{1}$ function of $\theta$ and $w$ in Case $\mathrm{b}$.

In our future work, we shall consider scalar functions, $f$, which are $C^{1}$ functions of $\theta$ and $w$ (see $(3.12 \mathrm{~b})$ ). For such functions, we find

$$
\begin{aligned}
& \frac{\partial f}{\partial \alpha}=-\frac{w}{\alpha-s} \frac{\partial f}{\partial w}, \\
& \frac{\partial f}{\partial z}=\frac{1}{\alpha-s} \frac{\partial f}{\partial w} .
\end{aligned}
$$

By use of $(3.12),(3.17 \mathrm{a}, \mathrm{b})$, we can define four operators $Q, P, M, L$ by

Definition 5.

$$
\begin{gathered}
\frac{\partial f}{\partial t} \equiv Q[f] \equiv \frac{(-\bar{a} w+\bar{b})}{\alpha-s} \frac{\partial f}{\partial w}, \\
\frac{\partial f}{\partial n} \equiv P[f] \equiv \frac{1}{\alpha-s} \frac{\partial f}{\partial \theta}, \\
\frac{\partial f}{\partial \bar{p}} \equiv M[f] \equiv-\frac{(\bar{b} w+\bar{a})}{\alpha-s} \frac{\partial f}{\partial w}, \\
L[f] \equiv b Q[f]+c P[f] .
\end{gathered}
$$


Now, we find the $(\alpha, \theta, z)$ coordinate form of (2.19), (2.24), (2.25), (2.33), (2.35) and (2.36)

Theorem 6. The System IIIb in $(\alpha, \theta, z)$ coordinates is

$$
\begin{gathered}
L[q]=\bar{q}, \quad L[S]=\bar{q}^{2}(K T)^{-1}, \\
L\left[\frac{\bar{q}}{K}\right]+\frac{C}{\rho^{2}} L[\rho]=-\frac{F \bar{q}^{2}}{T K}+G \bar{q}, \\
\rho L[b]=-\frac{\rho \bar{a} c^{2}}{\alpha-s}-A Q[\rho]-B Q[S]-C Q[q], \\
\frac{\rho c^{2} \bar{b}}{(\alpha-s)}+\frac{\rho c b}{\alpha-s}\left(\frac{\partial \psi}{\partial \theta}\right)=A M[\rho]+B M[S]+C M[q], \\
\frac{\rho b \bar{a}}{\alpha-s}-\rho b k+L[\rho]+\rho\{Q[b]+P[c]\}=0 .
\end{gathered}
$$

Proof. The proof follows by evaluating the terms of System IIIb by use of (3.12)(3.17).

For the vorticity vector in the special coordinates $(\alpha, \theta, z)$, we obtain

ThEOREM 7. The components of the vorticity vector are

$$
\begin{gathered}
\omega_{\alpha}=\bar{a}\left\{-M[c]-\frac{b}{\alpha-s} \frac{\partial \psi}{\partial \theta}\right\}+\bar{b}\{Q[c]-P[b]\}, \\
\omega_{z}=\bar{b}\left\{-M[c]-\frac{b}{\alpha-s} \frac{\partial \psi}{\partial \theta}\right\}-\bar{a}\{Q[c]-P[b]\}-\frac{c \bar{b}}{\alpha-s}, \\
\omega_{\theta}=M[b] .
\end{gathered}
$$

Proof. The above follows directly from (2.44)-(2.46) by use of (3.10), (3.12)-(3.16). As a check on the above calculations, we consider (3.18)-(3.22) for a plane equilibrium Prandtl-Meyer isentropic simple wave flow. In this case, we have for $S_{0}$ a constant

$$
\begin{gathered}
\bar{a}=1, \quad \bar{b}=0, \quad k=0 \\
K=\bar{q}=q=C=0, \quad S=S_{0}, \\
\frac{\partial b}{\partial z}=\frac{\partial c}{\partial z}=\frac{\partial \rho}{\partial z}=\frac{\partial b}{\partial \alpha}=\frac{\partial c}{\partial \alpha}=\frac{\partial \rho}{\partial \alpha}=0 .
\end{gathered}
$$

Thus, the operators $P, Q, M$ of definition 5 become

$$
Q[f]=M[f]=0, \quad P[f]=(\alpha-s)^{-1} \frac{d f}{d \theta}
$$

By use of (3.22), and (3.23) and the definition of $L[f]$ of (3.17), the relations (3.20), (3.22) become

$$
\begin{gathered}
\frac{d b}{d \theta}=-c, \\
\rho b+\frac{d}{d \theta}(\rho c)=0
\end{gathered}
$$


and (3.18), (3.21) vanish identically. However, (3.19) will be identically satisfied if and only if we consider that (3.19) has been obtained from another initial equation by division by $K$. The relation (3.25) is well known in Prandtl-Meyer flow (cf. p. 265 [2]); the relation (3.26) is an intrinsic form of the continuity equation. From (3.23a) it follows that the $(\alpha, \theta, z)$ components of the vorticity vanish or

$$
\omega^{i}=0 \text {. }
$$

The results of Tan [3] can be obtained by replacing the first relation of (3.23) by

$$
\bar{a}=\cos \psi, \quad \bar{b}=\sin \psi
$$

where $\psi$ is a $C^{1}$ function of $\theta$. Then, (3.20)-(3.22) becomes

$$
\begin{gathered}
d b / d \theta=-c \cos \psi, \\
-c \sin \psi=b d \psi / d \theta, \\
-\rho b \cos \psi=d(\rho c) / d \theta .
\end{gathered}
$$

The relations (3.29) and (3.30) are essentially those due to Tan (cf. (4.5), (4.6) [3]).

4. Simple waves. In a previous paper [5], we classified flows in relaxation (nonequilibrium) hydrodynamics into two classes: Class $\mathrm{I}, K$ is not constant and is a $C^{1}$ function of $\rho, S, q$ : Case II, $K$ is not constant and is a $C^{1}$ function of $x^{i}, t$. For simple waves in the $(x, t)$ plane, we showed that $K$ equal to a constant has almost all of the properties of simple waves of Case I. The basic property of this case and Case I (for simple waves) was that $c, b$ and all of the thermodynamical variables were $C^{1}$ functions of $\rho$. However, in Case II, the variables $c, b$ and all the thermodynamic variables, except $\bar{q}, K$, were $C^{1}$ functions of $\rho$. Also, for this case, $K / \bar{q}$ was a $C^{1}$ function of $\rho$.

At present, we shall not discuss all simple waves in three-dimensional steady flow. Instead we introduce three special classes of simple waves.

Definition 6. Simple waves of Case I satisfy the following three conditions: (1) the characteristic surfaces are a family of planes which envelope a right cylinder: (2) the bicharacteristics are a family of parallel lines in each characteristic plane; $(3) c, b$, $\psi\left(\right.$ see (3.11)) and all the thermodynamical variables are $C^{1}$ functions of $\rho$.

From the above definition and the fact that $\psi$ is defined in terms of the angle $\theta$ of the $(\alpha, \theta, z)$ coordinate system, we conclude that

Theorem 8. In simple waves of Case I, all the thermodynamic variables and $c, b, \psi$ depend on only $\theta$.

Now, we study (3.18)-(3.22) for simple waves of Case I. The operators $Q, P, L, M$ of (3.17) are given by (3.23). By direct computation, using (3.23) to evaluate the above operators and noting that $c, b$ and all of the thermodynamical variables are constant or $C^{1}$ functions of $\theta$,we find from the first equation of (3.18) that a constant $q_{0}$ exists so that

$$
\bar{q}=0, \quad q=q_{0} .
$$

From (4.1) and the second relation of (3.18) we see that a constant $S_{0}$ exists so that

$$
S=S_{0} \text {. }
$$

Further, (3.19) has $C^{1}$ solutions $\rho(\theta)$ if and only if: (1) $K$ does not vanish, $C$ goes to zero as $\bar{q}$ does; (2) $C / \bar{q}$ approaches a limit function, $* F(\rho)$. Then, (3.19) furnishes the following 
equation

$$
{ }^{*} F K^{2} d \rho / d \theta=\rho^{2} d K / d \theta .
$$

Finally, (3.20)-(3.22) imply the generalized Prandtl-Meyer relations (3.29)-(3.31). Thus, we obtain

Theorem 9. The only relaxation simple wave flows of Case I are (cf. p. 26 [5]) the equilibrium flows $\left(q=q_{0}\right)$ of constant entropy, propagating at the "frozen" speed $A$ (see (2.12)-(2.15)), which are generalized Prandtl-Meyer (that is, $\psi, b, c$ are solutions of (3.29)-(3.31). The relation (4.2) determines $K$ as a function of $\rho$.

In order to obtain a new class of simple waves, we introduce

Definition 7. The simple waves of Case IIa will satisfy properties 1, 2 of Case I but property 3 will be replaced by: $c, b, \psi$ and all of the thermodynamical variables, and $\bar{q} / K$ (but neither $\bar{q}$ nor $K$ ) will be $C^{1}$ functions of only $\rho$.

Since $\psi$ is a function of only $\theta$, it follows that, for Case IIa, the variables, $c, b$ and all of the thermodynamical variables except $K, \bar{q}$ are $C^{1}$ functions of only $\theta$. Thus, our present definition of Case IIa is an extension of the definition for simple waves of Case II for nonsteady one-dimensional flows (cf. p. 124 [5], and also Case a of definition 4).

Next, we verify

Theorem 10. Simple waves of Case IIa have the following properties:

(a) the ratio $K / \bar{q}$ is constant along any bicharacteristic;

(b) $K, q, S, \bar{q}, \rho$ are linked by three ordinary differential equations;

(c) $\rho, \psi, c, b$ satisfy the generalized Prandtl-Meyer relations (3.29)-(3.31).

Proof. From (3.17), we see that (3.18) leads to

$$
\begin{gathered}
c q^{\prime}=(\alpha-s) \bar{q}, \\
c K T S^{\prime}=(\alpha-s) \bar{q}^{2}
\end{gathered}
$$

where $q^{\prime}, S^{\prime}$ represent the derivatives of $q$, S, respectively, with respect to $\theta$. Dividing the equations (4.3), (4.4), we obtain

$$
K=\bar{q}^{*} G
$$

where

$$
{ }^{*} G(\theta)=q^{\prime} / T S^{\prime} .
$$

The relations (4.5) and (4.6) verify (a). It should be noted that a similar result is valid for simple waves in the $(x, t)$ plane (cf. Theorem 14 [5]).

When $f$ is $\rho$ or $\bar{q} / K$ then (3.17) becomes by use of (4.3), (4.5)

$$
\begin{aligned}
L[\rho] & =\frac{c \rho^{\prime}}{\alpha-s}, \\
L\left[\frac{\bar{q}}{K}\right] & =\frac{-{ }^{*} G^{\prime} c}{{ }^{*} G^{2}(\alpha-s)} .
\end{aligned}
$$

Thus. (3.19) reduces to

$$
\frac{\left({ }^{*} G\right.}{\rho^{2}} \rho^{\prime}+\left(\frac{F}{T}-G^{*} G\right) q^{\prime}-\frac{* G^{\prime}}{* G}=0 .
$$


From (4.3) and (4.4) and definition 7, it follows that $C^{1}$ functions $\bar{q}^{*}(\theta), K^{*}(\theta)$ exists so that

$$
\bar{q}^{*} \equiv(\alpha-s) \bar{q}, \quad K^{*} \equiv(\alpha-s) K
$$

The equations (4.3), (4.4) and (4.9) are the three ordinary differential equations linking $q, S, \rho, \bar{q}^{*}$. When $c, b, \bar{a}, \rho$ and the thermodynamical coefficients $F, T$ are known then (4.9) determines $q$ as a function of $\theta$, and (4.3) furnishes $\bar{q}^{*}$. For any $C^{1}$ function $S(\theta)$, the scalar $K^{*}$ is determined by (4.4).

The relations (3.20)-(3.22) lead to the generalized Prandtl-Meyer relations (3.29)(3.31) as

$$
\begin{aligned}
Q[\rho] & =Q[S]=Q[q]=0, \\
M[\rho] & =M[S]=M[q]=0 .
\end{aligned}
$$

In order to show how the previous theory may be used to classify simple waves, we introduce one additional type of very special simple wave.

Definition 8. Simple waves of Case IIb will satisfy property 1 of Case I but Properties 2 and 3 will be replaced by: the bicharacteristics form a family of radial lines in each characteristic plane; all the variables, and $\bar{q} / K$, but neither $\bar{q}$ nor $K$, will be $C^{1}$ functions of $\rho, S$ (cf. Case b of Definition 4).

By use of $(3.12 b),(3.14 c)$ we find that $k$ can be written as

$$
k=-1 /(\alpha-s)\left(1+w^{2}\right)^{1 / 2} .
$$

Theorem 11. Simple waves of Case IIb are generalized Prandtl-Meyer waves in which $c, b, \rho, \psi$ satisfy (cf. (3.29)-(3.31))

$$
\begin{gathered}
\partial b / \partial \theta=-c \cos \psi-(b / c)(-\bar{b} w+\bar{a}) \partial c / \partial w+C_{1} / \rho c \\
-c \sin \psi=b \partial \psi / \partial \theta-C_{2} / \rho c \\
\rho b \cos \psi-\frac{\rho b}{\left(1+w^{2}\right)^{1 / \overline{2}}}+\frac{\partial}{\partial \theta}(\rho c)+(-\bar{a} w+\bar{b})\left(\rho \frac{\partial b}{\partial w}-b \frac{\partial \rho}{\partial w}\right)=0
\end{gathered}
$$

where $C_{1}, C_{2}$ are $C^{1}$ function of $\theta, w$, which are arbitrary except for the algebraic condition

$$
-C_{1}(w \sin \psi+\cos \psi)=C_{2}(-w \cos \psi+\sin \psi) .
$$

Proof. By substituting (4.11), (3.17c) into (3.18) and (3.19) we obtain

$$
\begin{gathered}
b(-\bar{a} w+\bar{b}) \frac{\partial q}{\partial w}+c \frac{\partial q}{\partial \theta}=\bar{q}(\alpha-s), \\
b(-\bar{a} w+\bar{b}) \frac{\partial S}{\partial w}+c \frac{\partial S}{\partial \theta}=\bar{q}^{2}(K T)^{-1}(\alpha-s), \\
b(-\bar{a} w+\bar{b}) \frac{\partial(\bar{q} K)}{\partial w}+c \frac{\partial(\bar{q} / K)}{\partial \theta}+\frac{C}{\rho^{2}}\left[b(-\bar{a} w+b) \frac{\partial \rho}{\partial w}+c \frac{\partial \rho}{\partial \theta}\right] \\
+c \frac{\partial}{\partial \theta}(\bar{q} / K)=\left(\bar{q} G-\bar{q}^{2} F T^{-1} K^{-1}\right)(\alpha-s) .
\end{gathered}
$$

Since $\rho, S$ are independent, we can assume that they are $C^{1}$ functions of $\theta, \psi$ and that these variables are $C^{1}$ functions of $\theta, w$. Hence from (4.16)-(4.18), it follows that $C^{1}$ functions $\bar{q}^{*}$, 
$K^{*}$ exist so that

$$
\bar{q}=\bar{q}^{*} /(\alpha-s), \quad K=K^{*} /(\alpha-s)
$$

where $\tilde{q}^{*}, K^{*}$ are functions of only $\theta, w$. Then (4.16)-(4.18) are independent of $(\alpha-s)$, and consist of three partial differential equations for the five dependent variables $\rho$, $q, S, \bar{q}^{*}, K^{*}$. Further, from (2.12)-(2.15), the definition of $c, b$ in (2.17), and the condition that all the thermodynamic variables, except $\bar{q}, K$, are constant along a bicharacteristic, we see that $\rho, c, b$ are $C^{1}$ functions of only $\theta, w$. Hence, if $\rho, q, S, \bar{q}^{*}$, and $K^{*}$ satisfy (4.16)-(4.18), (4.20) where

$$
A \frac{\partial \rho}{\partial w}+B \frac{\partial q}{\partial w}+C \frac{\partial S}{\partial w}=C_{1}
$$

with $C_{1}$ a $C^{1}$ function of only $\theta, w$, and if $c, b, \psi, C_{2}$ are $C^{1}$ functions of only $\theta, w$, which satisfy (4.12)-(4.15), then (3.18)-(3.22) are satisfied and simple waves of Case IIb exist. It should be noted that (4.12)-(4.15), (4.16)-(4.18), (4.20) are eight equations (seven first order partial differential equations and one algebraic equation) for the ten unknowns $c, b, \psi, C_{1}, C_{2}, \rho, q, S, \bar{q}^{*}, K^{*}$. Hence, the system is undetermined and has two or more families of solutions.

An especially simple case arises when the characteristic planes are a family of parallel planes and the bicharacteristics are a family of radial lines of the circles in any charácteristic plane where the centers of these circles lie on a line parallel to the $x, y$ plane. In this case, the above family of algebraic and differential equations reduces to five first order ordinary differential equations (with $w$ as the independent variable) and three algebraic equations for the ten dependent variables $p, S, q$, etc.

Finally, we shall determine for what reacting fluids the above simple waves of Case I exist. Following the work of T. Y. Li [11], for the case of a simple dissociating gas, we assume that the specific enthalpy, $h$, and the pressure $p$, are known $C^{1}$ functions of $q, \rho, T$ and prove

Theогем 12. In the equilibrium steady state $(\bar{q}=0)$ of a chemically reacting fuid, if $h$ and $p$ are $C^{1}$ functions of $\rho, q, T$ such that the following Jacobian does not vanish

$$
J\left(\frac{\bar{q}, C}{p, T}\right)
$$

(where $C$ is defined by (2.8)) and

$$
\lim _{\bar{a} \rightarrow 0} \frac{C}{\bar{q}}={ }^{*} F
$$

exists, then simple waves of Case I exist.

Proof. If we assume that

$$
h=h(q, \rho, T), \quad p=p(q, \rho, T)
$$

are known $C^{1}$ functions of their arguments (for the simple dissociating gas, cf. p. 173 [11]), then the thermodynamic relation (cf. p. 170 [11] and pp. 5, 3 [12])

$$
T d S=d h-\frac{d p}{\rho}-\frac{\bar{q}}{K} d q
$$


becomes

$$
T d S=\bar{G} d q+\bar{F} d \rho+\bar{H} d T
$$

where

$$
\begin{gathered}
\bar{G} \equiv \frac{\partial h}{\partial q}-\frac{1}{q} \frac{\partial p}{\partial q}-\frac{\bar{q}}{K}, \\
\bar{F} \equiv \frac{\partial h}{\partial \rho}-\frac{1}{q} \frac{\partial p}{\partial \rho}, \\
\bar{H} \equiv \frac{\partial h}{\partial T}-\frac{1}{q} \frac{\partial p}{\partial T} .
\end{gathered}
$$

The condition $\bar{q}=0$ leads to a relation of the form (for a simple dissociating gas, cf. p. $173[11])$

$$
\bar{q}(\rho, q, T)=0
$$

which defines the equilibrium state. Further, the condition $C=0$ leads to

$$
\left.\left.\left.C \equiv \frac{\partial p}{\partial q}\right)_{S, \rho}=\frac{\partial p}{\partial T}\right)_{\rho, \rho}\left(\frac{\partial T}{\partial q}\right)_{S, \rho}+\frac{\partial p}{\partial q}\right)_{T, \rho}=0 .
$$

The coefficients

$$
\left.\left.\frac{\partial p}{\partial T}\right)_{\rho, e}, \quad \frac{\partial p}{\partial q}\right)_{T, \rho}
$$

can be computed from (4.21a). Also, if we assume $d S=d \rho=0$ in (4.22), we find

$$
\left.\frac{\partial T}{\partial q}\right)_{S, \rho}=-\frac{\bar{G}}{\bar{H}} .
$$

Thus, (4.25) leads to a relation of the form

$$
C(\rho, q, T)=0 \text {. }
$$

From (4.24), and (4.25), when $q$ is constant

$$
J\left(\frac{\bar{q}, C}{p, T}\right) \neq 0
$$

we can find $p, T$ as functions of $\rho$. Hence, in the equilibrium state $(\bar{q}=0)$ of a "proper" reacting fluid, one of the two basic conditions for the existence of simple waves of Case I is satisfied. The word "proper" implies that the functions of (4.21a) and (4.24) are known and (4.27) is satisfied. The limit function

$$
* F=\lim _{\bar{a} \rightarrow 0} \frac{C}{\bar{q}}
$$

must still be shown to exist.

In the case of the ideal simple dissociating gas of Lighthill [11], [13], the computations are easy and lead, for arbitrary constants $a, b, c, d$, to

$$
\begin{aligned}
& a \bar{q} \equiv q^{2}-(1-q) K \\
& C \equiv b \rho T+c \rho+d T \rho^{2}
\end{aligned}
$$


where $R_{2}, e^{*}$ are constants and $K$ is some known function of $p, q, T$. Evidently, for proper $K$, (4.27) will be valid and $* F$ will determine a function of $\rho$ (see (4.28)). Such a $K$ is $\left[q^{2} /(1-q)\right]+H(C, \rho, p)$ where $H$ is a differentiable function of $C, \rho, p$ so that when $C$ vanishes, $\partial H / \partial C$ is not zero, $H(C, \rho, p)$ is zero.

\section{REFERENCES}

1. N. Coburn, Intrinsic form of the characteristic relations in the steady supersonic flow of a compressible fluid, Quart. Appl. Math. 15, 237-248 (1957)

2. R. Courant and K. O. Friedrichs, Supersonic flow and shock waves, Interscience, New York 1948

3. F. Tan, Generalized Prandtl-Meyer flow, Technical Report No. DA-20-018-O.R.D.-17213, Detroit, Michigan, University of Michigan, Research Institute, Ann Arbor, Michigan, 1959

4. N. Coburn, Instrinsic form of the characteristic relations for a perfect compressible fluid in general relativity and non-steady Newtonian mechanics, J. Math. Mech. 8, 5 (1959)

5. N. Coburn, General theory of simple waves in relaxation hydrodynamics, J. Math. Anal. Appl. 11, $102-130$ (1965)

6. E. V. Stupochenko, and I. P. Stakhanov, The equations of relaxation hydrodynamics, Soviet Phys.Doklady, 4 (1960, Translated. Dokl. Akad. Nauk SSSR 134 (1960), 782-785

7. C. L. Dolph and N. Coburn, The method of characteristics in three-dimensional stationary supersonic flow of a compressible gas, Proc. Symposia Appl. Math., Vol. I, pp. 55-66, Amer. Math. Soc., Providence, R. I., 1949

8. N. Coburn, Vector and tensor analysis, Macmillan, New York, 1955

9. L. P. Eisenhart, Riemannian geometry, Princeton Univ. Press, Princeton, N. J., 1926

10. C. E. Weatherburn, Differential geometry of three dimensions, Cambridge Univ. Press, New York, 1927

11. T. Y. Li, Recent advances in nonequilibrium dissociating gasdynamics, A.R.S. Journal, February 1961

12. C. Yuan, Non-equilibrium hydrodynamics of a chemically reacting fluid, AT-ATOSR-20-63, University of Michigan Report, O.R.A. 05424 I-P, 1963

13. M. J. Lighthill, Dynamics of a dissociating gas. I. Equilibrium flow, J. Fluid Mech., 2, 1-32 (1957) 